\title{
Einfache Authentifikation?
}

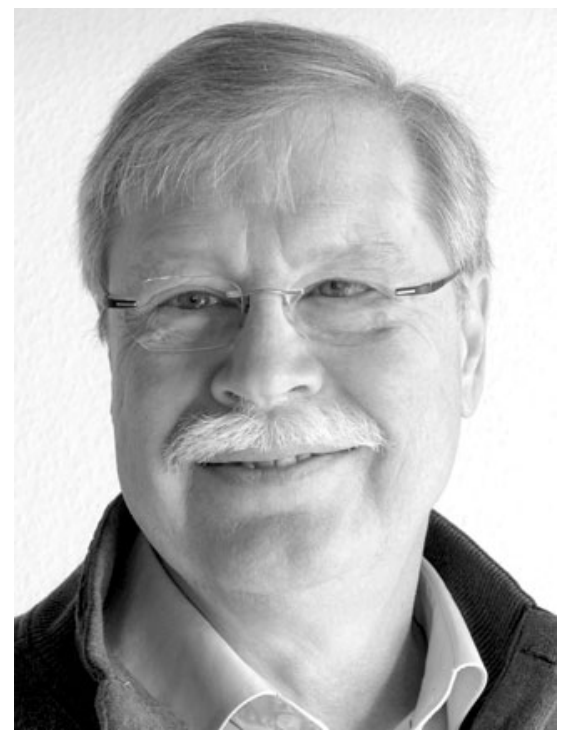

Ein Erfordernis für Authentifikationen gab es bereits vor der Einführung moderner digitaler Datenverarbeitungssysteme. Allerdings hat sich bereits in den Anfängen gezeigt, dass die genutzten Verfahren Schwächen hatten: die zum Zutritt in einen bestimmten Bereich erforderliche „Parole“ konnte leicht weitergegeben oder im wahrsten Sinne des Wortes abgehört werden oder Siegel auf Akkreditierungsschreiben konnten verfälscht werden. Sicherlich waren die erforderlichen Aufwände zwischen der Ausforschung einer Parole oder, um im heutigen Sprachgebrauch zu bleiben, eines Passworts und der Erstellung einer Kopie eines Siegels unterschiedlich hoch und erforderten zudem verschiedene handwerkliche Fertigkeiten. Das Hauptproblem lag aber darin, dass es ausreichte, dieses eine Authentifikationsmerkmal zu fälschen bzw. sich zu verschaffen, um weitere Vorteile zu erreichen.

Wie sieht dies in der heutigen, stark vernetzten, mobilen und digitalen Welt aus? Wer heute beispielsweise auf elektronischen Marktplätzen handelt oder einkauft, der trifft dort noch häufig auf Authentifizierungsmaßnahmen auf Grundlage einer User-ID in Verbindung mit einem Passwort. Einerseits wird moderne Technikzur Kommunikation und zum Handel verwendet, andererseits stammen die eingesetzten Authentifizierungsmechanismen aus dem Mittelalter. Dabei ist es nicht so, dass keine zur eingesetzten Technik passenden Alternativen existieren würden. Ihr Einsatz wird häufig durch Argumente wie fehlende Benutzerfreundlichkeit oder zu hohe Kosten gebremst. Diese Meinung ändert sich seitens der Anbieter spätestens dann, wenn Systemschäden zunehmen bzw. Kosten aufgrund erhöhter Reklamationsbearbeitung überhandnehmen.

Dabei reagieren wir als Kunden besonders sensibel, wenn wir eine Finanztransaktion freigeben sollen. So stellen wir uns u.a. die Frage, ob sichergestellt ist, dass niemand anderes unsere elektronische Identität nutzen kann, um Geld von unserem Konto abzubuchen. Die im Mai letzten Jahres veröffentlichten „Mindestanforderungen an die Sicherheit von Internetzahlungen" (kurz MaSI) der BaFin fordern eine starke (gemeint ist eine 2-Faktor-) Kundenauthentifizierung. Die kommende europäische Zahlungsverkehrsdiensterichtlinie PSD 2 fordert darüber hinaus zusätzlich, auch Transaktionsdaten mit dem Authentifikationsverfahren abzusichern. Konkrete Verfahren werden nicht vorgegeben. Im vorliegenden Heft wird daher das Thema der Multifaktor-Authentifizierung aus verschiedenen Richtungen beleuchtet.

Stephan Spitz gibt in seinem Beitrag einen Überblick über verschiedene Authentifizierungsprotokolle einschließlich eines Ausblicks auf zu betrachtende Sicherheitsfragen bei der Implementierung in mobilen Endgeräten.

Jens Bender und Dennis Kügler versuchen in ihrem Artikel ergänzend die Frage zu beantworten, was eigentlich eine starke Authentisierung ausmacht.

Der Beitrag von Gunnar Jacobsen widmet sich dann konkret mit der für einen Endezu-Ende verschlüsselten transparenten Datenaustausch per E-Mail erforderliche Schlüssel- und Zertifikatverteilung.

Markus Hertlein, Pascal Manares und Norbert Pohlmann stellen ihr Konzept vor, welches Handhabbarkeit, einfache Integration und (optional um Hardware Token erweiterbare) Sicherheit der Mehrfaktor-Authentifizierung mit dem Smartphone verspricht. Hannes Tschofenig untersucht die Authentikationsprobleme für das Internet der Dinge und gibt Hinweise, wie mit existierenden Technologien die Sicherheitsanforderungen gelöst werden könnten. Die aufgrund der Vielzahl der entstandenen Authentifikationssysteme auftretenden Interoperabilitätsprobleme zwischen den für starke Authentifikation eingesetzten Geräten will die FIDO Allianz beseitigen, deren Konzepte Rolf Lindemann mit existierenden Mehr-Faktor-Authentikationssystemen vergleicht.

Die Nutzung von Serversignaturen gemäß der neuen eIDAS Verordnung, die der handschriftlichen Unterschrift in Europa gleichgestellt ist und die dort formulierten Anforderung an die Authentifikationsverfahren werden von Kim Nguyen et al. untersucht. Sandro Amendola und Detlef Kraus beschreiben in ihrem Artikel den aktuellen Stand der aufsichtsrechtlichen Regulierungsanforderungen zur starken Authentifizierung und versuchen die Frage zu beantworten, ob hiermit tatsächlich Schäden verhindert oder reduziert werden können.

Detlef Kraus, Helmut Reimer 